13.2

\title{
Взаимодействие молекулярных ионов кислорода с поверхностью меди: особенности энергетических спектров распыленных и обратнорассеянных ионов
}

\author{
(C) A.Б. Толстогузов ${ }^{1-3}$, C.И. Гусев ${ }^{1}$, D.J. Fu ${ }^{3}$ \\ ${ }^{1}$ Рязанский государственный радиотехнический университет им. В.Ф. Уткина, Рязань, Россия \\ ${ }^{2}$ Centre for Physics and Technological Research, Universidade Nova de Lisboa, Caparica, Portugal \\ ${ }^{3}$ Key Laboratory of Artificial Micro- and Nanostructures of Ministry of Education and Hubei Key Laboratory \\ of Nuclear Solid Physics, School of Physics and Technology, Wuhan University, Wuhan, China \\ E-mail: a.tolstoguzov@fct.unl.pt
}

Поступило в Редакцию 22 ноября 2021 г.

В окончательной редакции 2 февраля 2022 г.

Принято к публикации 2 февраля 2022 г.

\begin{abstract}
Проведено экспериментальное исследование энергетических спектров ионов, эмитированных с поверхности поликристаллической медной мишени при ее облучении молекулярными ионами $\mathrm{O}_{2}^{+}$с энергией $2 \mathrm{keV}$. Среди положительно заряженных вторичных ионов превалировали распыленные атомные ионы $\mathrm{Cu}^{+}$и $\mathrm{O}^{+}$, a среди отрицательных ионов помимо распыленных ионов $\mathrm{Cu}^{-}, \mathrm{CuO}_{2}^{-}, \mathrm{O}^{-}$и $\mathrm{O}_{2}^{-}$были зарегистрированы довольно интенсивные упруго и неупруго обратнорассеянные ионы $\mathrm{O}^{-}$, образованные при перезарядке бомбардирующих ионов $\mathrm{O}_{2}^{+}$.
\end{abstract}

Ключевые слова: энергетические спектры, ионное распыление, обратнорассеянные ионы, перезарядка.

DOI: 10.21883/PJTF.2022.07.52294.19088

Взаимодействие низкоэнергетических ионов и атомов кислорода с поверхностью твердых тел сопровождается рядом процессов, среди которых можно выделить распыление (травление) бомбардируемой мишени и рассеяние ионов кислорода. Распыление ионами $\mathrm{O}_{2}^{+}$успешно используется в методе масс-спектрометрии вторичных ионов (secondary ion mass spectrometry, SIMS [1]) для повышения чувствительности метода за счет увеличения ионного выхода электроположительных примесей. Что касается низкоэнергетического ионного рассеяния (lowenergy ion scattering, LEIS [2,3]), то здесь преимущество отдается ионам инертных газов $\left(\mathrm{He}^{+}, \mathrm{Ne}^{+}\right)$, обеспечивающим уникальную чувствительность к составу и структуре самого верхнего слоя, а также ионам щелочных металлов $\left(\mathrm{Na}^{+}, \mathrm{Cs}^{+}\right)$, позволяющим провести количественный элементный анализ поверхности. Рассеяние атомных и ионных пучков кислорода от поверхности металлов $\mathrm{Mg}$, $\mathrm{Al}$ и $\mathrm{Ag}$ [4], полупроводниковых кристаллов $\mathrm{Si}$ [5], а также ионных кристаллов LiF [6] интенсивно изучалось в конце 90 -х годов. В основном это были теоретические исследования, направленные на выяснение механизмов образования отрицательных ионов кислорода (перезарядки), а эксперименты проводились при скользящих углах падения и малых углах рассеяния, не превышающих $40^{\circ}$, что увеличивало время взаимодействия бомбардирующих частиц с атомами поверхности. Теоретический и практический интерес к процессам образования отрицательных ионов кислорода, стимулированный перспективами создания эффективных источников отрицательных ионов, по-прежнему существует (см., например, работу [7] и ссылки в ней).
Цель настоящей работы состоит в экспериментальном исследовании особенностей энергетических спектров распыленных и рассеянных ионов, сформированных при бомбардировке поверхности поликристаллической медной мишени молекулярными ионами ${ }^{16} \mathrm{O}_{2}^{+}$с энергией $2 \mathrm{keV}$ ( $1 \mathrm{keV} /$ atom). При этом практически одновременно измерялись спектры как положительных, так и отрицательных распыленных и обратнорассеянных ионов в направлении, перпендикулярном поверхности мишени.

В качестве мишени использовались полированные образцы высокочистой поликристаллической меди, а экспериментальные исследования проводились в Istituto per l'Energetica e le Interfasi (CNR-IENI, Padua, Italy) на высоковакуумной многофункциональной установке, оснащенной квадрупольным масс-энергоанализатором Hiden EQS 1000 и дуоплазматронным источником ионов DP50B (VG Fison). Детальное описание установки можно найти в [8], а геометрия эксперимента схематично представлена на рис. 1. Основная особенность установки - это возможность измерения масс-спектров (по отношению массы ионов к заряду $M / q$ при фиксированной энергии) и энергетических спектров (при фиксированном значении $M / q$ ) для ионов обеих полярностей в узком телесном угле $\Delta \sim 10^{-4} \mathrm{sr}$ при отсутствии электрического поля в пространстве между поверхностью мишени и входным электродом масс-энергоанализатора. Источник ионов был оснащен масс-фильтром Вина, и сфокусированный ионный пучок ${ }^{16} \mathrm{O}_{2}^{+}$с плотностью тока менее $100 \mu \mathrm{A} / \mathrm{cm}^{2}$ разворачивался на поверхности образца в растр размером $200 \times 200 \mu \mathrm{m}$. Угол падения зондирующих ионов относительно поверхности образ- 


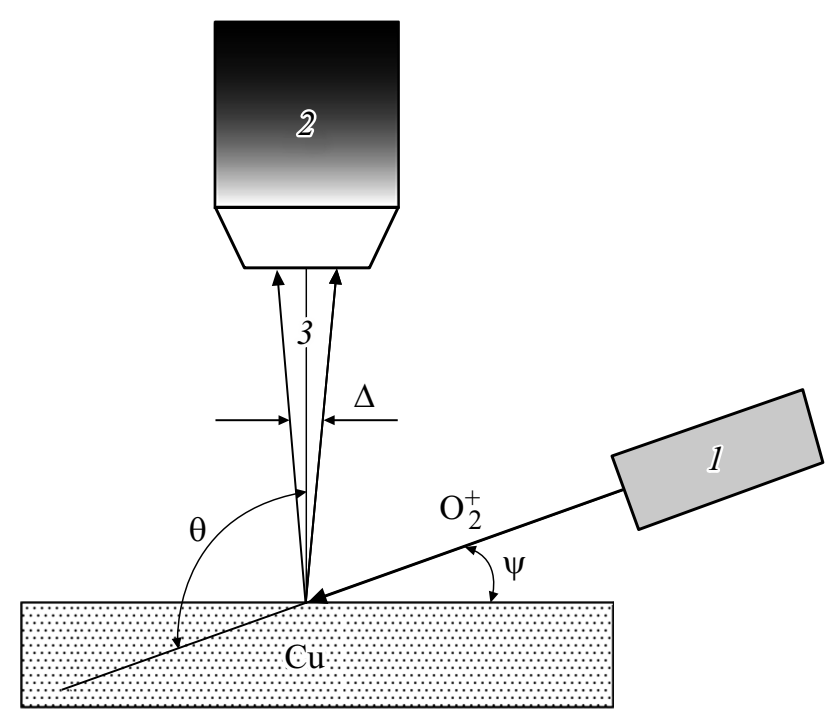

Рис. 1. Схематическое изображение геометрии эксперимента. 1 - источник зондирующих ионов, 2 - массэнергоанализатор, 3 - пучок анализируемых ионов. Угол падения зондирующего пучка $\psi=30^{\circ}$, угол рассеяния $\theta=120^{\circ}$, угол входа ионов в масс-энергоанализатор $\Delta \sim 10^{-4} \mathrm{sr}$.

ца $\psi$ был равен $30^{\circ}$, а угол рассеяния $\theta$, т.е. угол между направлением движения бомбардирующих ионов и осью масс-энергоанализатора, составлял $120^{\circ}$ (рис. 1). Измерения проводились в стационарных (steady-state) условиях, когда интенсивность энергетических спектров не зависела от времени бомбардировки (дозы бомбардирующих ионов). Вакуум в аналитической камере в процессе измерений был не хуже $10^{-7} \mathrm{~Pa}$.

На рис. 2 в полулогарифмическом масштабе представлены энергетические спектры наиболее интенсивных положительных и отрицательных вторичных ионов, содержащих медь и кислород. Отметим, что шкала интенсивности на рис. 2 не корректировалась на разную эффективность детектирования положительных и отрицательных ионов вторично-электронным умножителем. Максимумы всех спектров на рис. 2, $a$ были расположены в диапазоне $1-3 \mathrm{eV}$, но из-за большой интенсивности для ионов ${ }^{63} \mathrm{Cu}^{+}$наблюдались насыщение и перегрузка детектора, что приводило к провалу пика этого спектра. Спектр положительно заряженных ионов ${ }^{63} \mathrm{Cu}^{+}$- это (квази)классический спектр распыленных частиц, который может быть описан формулой Томпсона [9] в рамках модели линейных каскадов упругих и неупругих соударений, инициированных бомбардирующими ионами в приповерхностной области мишени. Не вдаваясь в детали процессов ионизации распыленных частиц (см., например, работу [10] и ссылки в ней), можно предположить, что протяженный „хвост“ этого спектра был сформирован за счет передачи энергии распыленным частицам меди от быстрых обратнорассеянных (backscattering) атомов/ионов кислорода и атомов/ионов отдачи (direct-recoil) меди. Спектры отрица- тельных атомных $\left({ }^{63} \mathrm{Cu}^{-}\right)$и молекулярных $\left({ }^{63} \mathrm{Cu}^{16} \mathrm{O}_{2}^{-}\right)$ ионов, представленные на том же рисунке, отличаются от спектра положительных ионов меди. Они более узкие, и их интенсивность спадает до уровня шумового сигнала вторично-электронного умножителя при энергиях выше $20 \mathrm{eV}$. Вероятнее всего, формирование отрицательных ионов происходит за счет процессов электронного обмена в очень тонком слое оксидов меди, который постоянно образуется (возобновляется) на поверхности мишени и распыляется бомбардирующими ионами кислорода. Согласно данным SRIM-2008 [11], глубина внедрения (projected range) для ионов $1 \mathrm{keV}{ }^{16} \mathrm{O}$, т.е. глубина, при которой концентрация имплантированных ионов кислорода в меди достигает максимума, составляет примерно $1 \mathrm{~nm}$ при данной геометрии эксперимента. С учетом того, что электронное сродство для меди имеет отрицательное значение $(-1.9 \mathrm{eV}[12])$, процесс образования отрицательных ионов меди при отсутствии на поверхности медной мишени электроположительных элементов типа Cs, которые могут понизить работу выхода мишени, должен быть эндотермическим, т.е. идти с поглощением энергии.

На рис. 2, $b$ представлены энергетические спектры атомных и молекулярных ионов кислорода обеих полярностей. Спектр ${ }^{16} \mathrm{O}^{+}$по форме напоминает спектр ${ }^{63} \mathrm{Cu}^{-}$с той лишь разницей, что его максимум сдвинут в сторону бо́льших энергий $(\sim 8-10 \mathrm{eV})$ и он является более протяженным (простирается вплоть до энергий порядка 80-100 eV). Примерно также выглядит спектр отрицательных молекулярных ионов кислорода ${ }^{16} \mathrm{O}_{2}^{-}$, представленный на том же рисунке. Можно предположить, что формирование положительных атомных и отрицательных молекулярных ионов кислорода происходит в поверхностном слое оксидов меди так же, как и отрицательных ионов меди и диоксида меди, рассмотренных выше.

Следует обратить внимание на существенную разницу в спектрах положительных и отрицательных атомных ионов кислорода (рис. 2, $b$ ). По нашему мнению, энергетический спектр ${ }^{16} \mathrm{O}^{-}$представляет собой суперпозицию двух спектров, а именно спектра распыленных ионов кислорода с максимумом при энергиях около $10 \mathrm{eV}$ и спектра упруго и неупруго обратнорассеянных ионов кислорода с максимумом в диапазоне энергий 450-460 eV. Положение этого максимума можно оценить в рамках модели упругих парных соударений [2]:

$$
E_{1}=\left(\frac{\cos \theta+\sqrt{\alpha^{2}-\sin ^{2} \theta}}{1+\alpha}\right)^{2} E_{0}=K E_{0},
$$

где $E_{0}$ и $E_{1}-$ энергии бомбардирующих и обратнорассеянных ионов соответственно, $\alpha=M_{1} / M_{0}$ - отношение масс атома поверхности $(\mathrm{Cu})$ и первичного иона $\left({ }^{16} \mathrm{O}\right)$, $K$ - кинетический фактор. В условиях нашего эксперимента $\left(\theta=120^{\circ}, \alpha=3.97\right)$ кинетический фактор равен $\sim 0.46$, что примерно соответствует на шкале энергий максимуму энергетического спектра ионов ${ }^{16} \mathrm{O}^{-}$. 

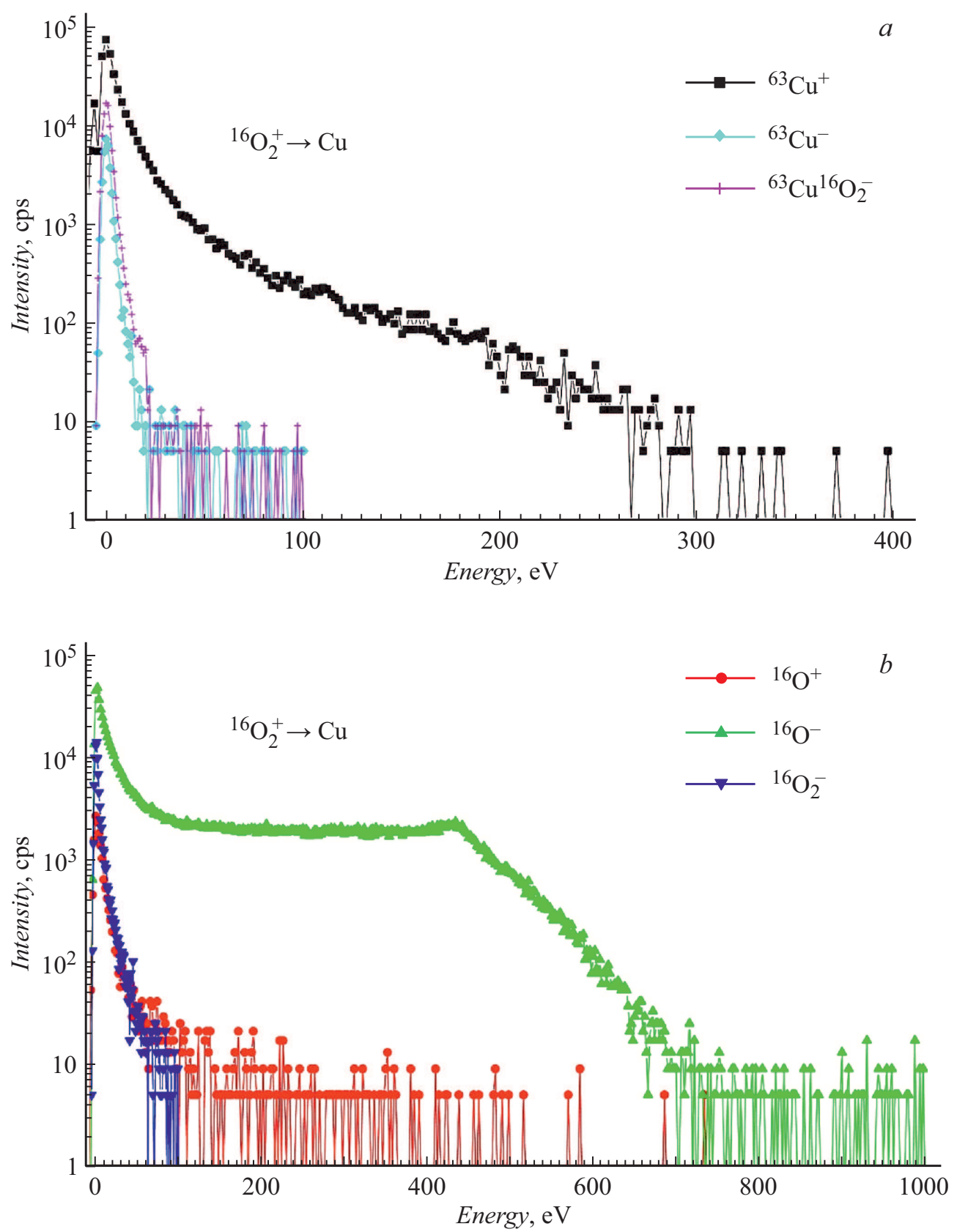

Рис. 2. Энергетические спектры: $a$ - распыленных ионов, содержащих медь; $b-$ распыленных $\left({ }^{16} \mathrm{O}^{+},{ }^{16} \mathrm{O}^{-}{ }^{1}{ }^{16} \mathrm{O}_{2}^{-}\right)$и рассеянных $\left({ }^{16} \mathrm{O}^{-}\right)$ионов кислорода.

Таким образом, при бомбардировке поверхности меди положительно заряженными молекулярными ионами кислорода происходит одновременно несколько взаимосвязанных процессов: 1) распыление материала мишени, сопровождаемое эмиссией положительно и отрицательно заряженных ионов меди; 2) распыление из поверхностного ионно-индуцированного слоя оксидов меди кислородсодержащих атомов и молекул, сопровождаемое образованием ионов обеих полярностей; 3) интенсивное упругое и неупругое рассеяние бомбардирующих ионов кислорода, сопровождаемое процессами перезарядки, т. е. образованием отрицательных атомных ионов кислорода за счет возможности многоканального захвата электронов из валентной зоны и зоны проводимости меди. Важно отметить, что рассеяние положительных ионов кислорода на уровне чувствительности нашей аппаратуры не наблюдалось.

\section{Благодарности}

Один из авторов (А.Б. Толстогузов) выражает благодарность C. Pagura (CNR-IENI, Padua, Italy) за возможность проведения экспериментальных исследований в его лаборатории. 


\section{Финансирование работы}

Работа выполнена в рамках государственного задания Министерства науки и высшего образования РФ (FSSN-2020-0003).

\section{Конфликт интересов}

Авторы заявляют, что у них нет конфликта интересов.

\section{Список литературы}

[1] A. Benninghoven, F.G. Rüdenauer, H.W. Werner, Secondary ion mass spectrometry: basic concepts, instrumental aspects, applications, and trends (Wiley, N.Y., 1987).

[2] Е.С. Машкова, В.А. Молчанов, Применение рассеяния ионов для анализа твердых тел (Энергоатомиздат, М., 1995).

[3] H. Brongersma, M. Draxler, M. de Ridder, P. Bauer, Surf. Sci. Rep., 62 (3), 63 (2007). DOI: 10.1016/j.surfrep.2006.12.002

[4] M. Maazouz, L. Guillemot, T. Schlatholter, S. Ustaze, V.A. Esaulov, Nucl. Instrum. Meth. Phys. Res. B, 125 (1-4), 283 (1997). DOI: 10.1016/S0168-583X(96)00807-5

[5] M. Maazouz, L. Guillemot, V.A. Esaulov, D.J. O'Connor, Surf. Sci., 398 (1-2), 49 (1998). DOI: $10.1016 / \mathrm{S} 0039-6028(98) 80010-1$

[6] C. Auth, A.G. Borisov, H. Winter, Phys. Rev. Lett., 75 (12), 2292 (1995). DOI: 10.1103/PhysRevLett.75.2292

[7] Z. Zong, H. Zhou, B. Jin, X. Zhang, G. Wang, L. Zhou, X. Chen, J. Phys. Chem. C, 124 (33), 18054 (2020). DOI: $10.1021 /$ acs.jpcc.0c04040

[8] A. Tolstogouzov, S. Daolio, C. Pagura, C.L. Greenwood, Int. J. Mass Spectrom., 214 (3), 327 (2002). DOI: $10.1016 / \mathrm{S} 1387-3806(02) 00523-7$

[9] M.W. Thompson, Phil. Mag., 18 (152), 377 (1968) DOI: $10.1080 / 14786436808227358$

[10] Sputtering by particles bombardment. Issue III. Characterization of sputtered particles, technical applications, ed. by R. Behrisch, K. Wittmaack (Springer-Verlag, Berlin, 1991).

[11] http://www.srim.org/ (дата обращения 20.11.2021).

[12] https://www.nuclear-power.com/copper-affinity-electronegativity-ionization/ (дата обращения 20.11.2021). 